\title{
Role of Nitrofurantoin in the Management of Urinary Tract Infection - A Systematic Review
}

\author{
Md. Shamshir Alam ${ }^{1}$, Komal Rana ${ }^{2}$, Shweta Bhardwaj ${ }^{3}$, Jagatheesh Kaliaperumal ${ }^{4}$, Md. Sarfaraj Hussain ${ }^{5}$, Amit Mittal ${ }^{6}$ \\ 1Department of Pharmacy Practice, MM College of Pharmacy, Maharishi Markandeshwar (Deemed to be University), \\ Mullana-Ambala, Haryana, India. ${ }^{2}$ Department of Pharmacy Practice, MM College of Pharmacy, Maharishi \\ Markandeshwar (Deemed to be University), Mullana-Ambala, Haryana, India. ${ }^{3}$ Department of Pharmacy Practice, MM \\ College of Pharmacy, Maharishi Markandeshwar (Deemed to be University), Mullana-Ambala, Haryana, India. \\ 4Department of Pharmacology, MM College of Pharmacy, Maharishi Markandeshwar (Deemed to be University), \\ Mullana-Ambala, Haryana, India. ${ }^{5}$ Department of Pharmacognosy, RV Northland Institute of Pharmacy, Gautam Budh \\ Nagar, Uttar Pradesh, India. ${ }^{6}$ Department of Radiodiagnosis, MMIMSR, M. M. Deemed to be University, Mullana, \\ Haryana, India.
}

\section{ABSTRACT}

\section{BACKGROUND}

Urinary tract infections are often caused by bacteria resistant to antibiotics which have evolved to be a major health issue in recent years. Often, urinary tract infections are very hard to treat and if not appropriately treated in time, may result in serious consequences, particularly, when it spreads to kidneys ultimately resulting in an increase in morbidity and mortality. Nitrofurantoin, a first line agent and has bactericidal action, frequently used to treat uncomplicated urinary tract infection. The literature was searched with published sources from Medline, PubMed and Embase search engines. Published articles were searched, of which 81 articles were eligible to be included for this systematic review. Nitrofurantoin is reduced by the action of bacterial flavoproteins to reactive intermediate compounds that non-specifically inactivate ribosomal proteins resulting in inhibition of protein synthesis. Various mechanisms seem to be responsible for the reduced capability of microorganism to acquire resistance in a faster manner. Nitrofurantoin exhibits high quality success against most bacteria anticipated in urinary tract infection. Nitrofurantoin has been recommended for prophylaxis in the treatment of reinfection in case of recurrent uncomplicated urinary tract infections in many western countries. Nitrofurantoin is one of the treatment options for urinary tract infection due to extended spectrum beta lactamase producing Escherichia coli. In pregnant women with urinary tract infection, nitrofurantoin can be appropriate treatment. Also, nitrofurantoin associated reactions have been reported in many studies. This review updates the clinical use of nitrofurantoin, including new facts about the role of nitrofurantoin in the therapy of community acquired urinary tract infection, adverse outcomes, complications, interactions and antibiotic resistance mechanism against different uropathogens.

\section{KEY WORDS}

Nitrofurantoin, Drug Information Review, Urinary Tract Infection, Uncomplicated, Clinical Manifestations

\author{
Corresponding Author: \\ Dr. Md. Shamshir Alam, \\ Associate Professor, \\ Department of Pharmacy Practice, \\ MM College of Pharmacy, \\ Maharishi Markandeshwar \\ (Deemed to be University), \\ Mullana-Ambala-133207, \\ Haryana, India. \\ E-mail: shamshir_pharma@yahoo.com
}

DOI: $10.14260 /$ jemds $/ 2019 / 824$

Financial or Other Competing Interests: None.

How to Cite This Article: Alam MS, Rana K, Bhardwaj S, et al. Role of nitrofurantoin in the management of urinary tract infection- a systematic review. J. Evolution Med. Dent. Sci. 2019;8(50):3805-3812, DOI: $10.14260 /$ jemds $/ 2019 / 824$

Submission 17-09-2019,

Peer Review 26-11-2019,

Acceptance 03-12-2019,

Published 16-12-2019. 


\section{BACKGROUND}

Presence of bacteria in the urine is defined as urinary tract infection (UTI), pathologically termed as bacteriuria. UTI are a notable cause of impairments in infants, boys, older men and females of all ages. ${ }^{1}$ UTI were first reported in Egypt in $1550 \mathrm{BC}$, and are still among the most prevalent bacterial infections around the world. The prevalence of UTI appears to be a J-shaped distribution, with higher frequency between very young children, which gradually increases with age. It is affects 150 million people each year worldwide, with an annual incidence of $12.6 \%$ in women and $3 \%$ in men. UTI is one of the major experienced problems in urological clinics. ${ }^{2}$

UTI are classified into lower urinary tract infection known as cystitis and upper UTI known as pyelonephritis. ${ }^{3}$. Common symptoms of lower UTI, cystitis (affecting the urethra and urinary bladder) include frequent urination, burning sensation and pain during urination (dysuria), suprapubic pain and/or lower abdominal discomfort, and cloudy and/or bloody urine with usually foul smell. UTI is mainly affecting the ureters and the kidneys that are often identified by the presence of bacteriuria and pyuria accompanied by flank pain and fever. ${ }^{3}$

Escherichia coli (Escherichia coli) are the predominant cause of UTI. It is a Gram-negative bacterium, whose primary place is the large bowel in case of vertebral animals, is responsible for approximately $80 \%$ of community acquired infections, and $25 \%$ of nosocomial infections. Staphylococcus saprophyticus is retrieved in 10 to $15 \%$ of community acquired infections, followed by Klebsiella species, Enterobacter, Proteus and Enterococcus species. ${ }^{4}$

The treatment goal of UTI includes complete eradication of the microorganism from the host body. The therapy of UTI mainly includes antimicrobial agent. Nitrofurantoin is a synthetic chemotherapeutic agent belongs to nitro furan family, was first discovered in 1940s. It was derived from furan by addition of a nitro group and a side chain containing hydantoin. It was first initiated into health care practice in $1952.5^{5}$ In 1953, it is accepted by US FDA for the therapy of lower UTI. ${ }^{6}$ The solubility of nitrofurantoin being a weak acid is altered by pH. ${ }^{5}$ Nitrofurantoin is mostly clinically active against a wide range of Gram positive and Gram negative bacteria, including many strains of common urinary tract pathogens. Nitrofurantoin is primarily used as an oral antibacterial agent for the treatment of genitourinary infections. $^{7}$ Nitrofurantoin, a broad-spectrum antibiotic indicated for UTIs. It is mainly indicated for prophylactic setting in the patients with recurrent UTIs.

\section{METHODS}

The literature was searched with published sources from Medline, PubMed and Embase search engines, of the searched items 81 articles were eligible to be included for this systematic review. All relevant articles in English published during 1978 and 2018 were retrieved, checked for suitability and included in this review. The keywords used included UTI, UTI epidemiology, UTI symptoms, nitrofurantoin in UTI, nitrofurantoin mechanism of action, UTI treatment and nitrofurantoin resistance in UTI.
Research articles, review articles or meta-analyses and guidelines, accommodating particulars about prevalence, symptoms, risk factors, mechanisms of action, antimicrobial treatment and antimicrobial resistance were included in this present review. Selected articles were identified through careful understanding of the study title and the theoretical aspect of every article picked out. The full articles were obtained by means of various resources. All the selected articles were thoroughly studied and required data were extracted out which is transferred into computer excel sheet, evaluated and compiled in the form of present review.

\section{RESULTS}

\section{Pharmacokinetics \& Pharmacodynamics}

Absorption- Gastrointestinal tract (GIT) is the primary site for the absorption of nitrofurantoin. The absorption process takes place in the proximal part of small intestine. The amount of drug absorbed and the duration of therapeutic urinary concentrations was significantly increased, when nitrofurantoin was given with food. ${ }^{8}$ Particle size affects bioavailability of nitrofurantoin, due to this reason the microcrystalline form gets slowly absorbed in the gastrointestinal tract and is excreted more slowly in the urine as compare to the microcrystalline form. The decreased rate of absorption of nitrofurantoin in GIT in microcrystalline form results in decreased rates of incidence of nausea and vomiting. ${ }^{5}$ As the absorption of nitrofurantoin through gastrointestinal route is adequate, the effect on the intestinal flora is minimum. ${ }^{9}$

Distribution- Nitrofurantoin gets easily absorbed and quickly distributed into most of the body fluids. It is rapidly excreted in large amounts in bile and urine. With the irregularity of the active drug secretion in the kidney tubules and biliary drug transport, nitrofurantoin crosses across body membranes through diffusion. ${ }^{10}$ Therapeutic concentration of parenterally administered nitrofurantoin were not adequate in aqueous humour, cerebrospinal fluid, prostatic secretions, amniotic fluid and umbilical cord serum, either in man nor in animal models. ${ }^{5}$

Elimination- Nitrofurantoin is primarily eliminated through biliary means and urinary excretion (Unchanged nitrofurantoin) and enzymatic degradation. ${ }^{10}$ Around 25\% of nitrofurantoin is excreted through renal route and some is eliminated through the gastrointestinal tract. The bioavailability of nitrofurantoin is almost $90 \%$, however the plasma concentrations are very low $(<1 \mathrm{mg} / \mathrm{L}$ after an oral dose of $100 \mathrm{mg}$ ). The elimination half-life is short, generally around 1 hour, and $27-50 \%$ of the drug is excreted unchanged in the urine. Although peak urinary levels are $>100 \mathrm{mg} / \mathrm{L}$ (range 50-200 mg/L), however these are maintained for a relatively short period of time.11,12

Excretion in Patients with Impaired Function- In the condition of compromised renal function, the levels of nitrofurantoin concentration drop in urine below the therapeutic concentration range, however, the serum levels 
increase to the toxic range. Thus, its efficacy is compromised in patients of renal impairment, with an associated higher risk of toxic and adverse effects. ${ }^{13}$

Pharmacodynamics- Nitrofurantoin exerts bactericidal action against receptive pathogens. As nitrofurantoin shows timedependent phenomena, with $\mathrm{T}>\mathrm{MIC}$, being the pharmacokinetic-pharmacodynamic parameter, suitably coordinate with efficacy; therefore, maintenance of plasma concentration higher than the required MIC value for a relatively long-time post-dose is required. As a consequence, there might be a risk of treatment failure for those microorganisms with a higher MIC value, however, the sensitivity threshold of $64 \mathrm{mg} / \mathrm{L}$ for urinary pathogens still seems too high. ${ }^{14}$

Doses- The conventional oral dose of nitrofurantoin therapy for UTI patient is recommended in the range between 50-100 mg four times a day. ${ }^{8}$ It is rapidly absorbed and quickly eliminated through renal route. Plasma concentrations of nitrofurantoin are generally very low, $<0.6 \mathrm{mg} / \mathrm{L}^{15}$, but high concentrations of nitrofurantoin (up to $250 \mathrm{mg} / \mathrm{L}$ ) have been reported in urine. In most instances the bioavailability of nitrofurantoin is high, except in patients with severe renal failure, where serum concentrations are almost negligible. ${ }^{14,16}$

Bacterial Spectrum- Nitrofurantoin is active against most common uropathogens such as Escherichia coli, Citrobacter species, Staphylococcus saprophyticus, and Enterococcus species. Enterobacter and Klebsiella species are only moderately inhibited by nitrofurantoin therapy, while Proteus species, Providencia species, Morganella morganii, Serratia species, Pseudomonas species, and Acinetobacter species are mainly resistant to nitrofurantoin.17,18 Nitrofurantoin is highly effective against both Escherichia coli and $S$ saprophyticus. For these pathogens, under stationary conditions a bactericidal effect (defined as a $>3$ log decrease in $\mathrm{cfu} / \mathrm{mL}$ ) was obtained within 4-6 hours and the maximum antibacterial activity was obtained at $8 \times$ MIC. A bactericidal effect might be observed after 12-24 hours at a concentrations of $16 \times$ MIC or higher (i.e. $>64 \mathrm{mg} / \mathrm{L}$ for vancomycin-susceptible Escherichia faecium (E faecium) and $>128 \mathrm{mg} / \mathrm{L}$ for vancomycin-resistant $E$ faecium. ${ }^{14}$ Nitrofurantoin is beneficial against Escherichia coli and Enterococci related nosocomial lower UTIs (catheterassociated). Nitrofurantoin is active against most strains of multidrug resistant Gram-negative bacilli, including extended spectrum beta lactamase-producing bacteria. ${ }^{18}$ Nitrofurantoin is also active against both vancomycin sensitive as well as resistant enterococci. ${ }^{18,19}$

Mechanism of Action- Nitrofurantoin exhibits types of mechanisms of action, which are not fully understood. Intracellular nitroreductases, a household of evolutionarily related proteins, produce the active form of the drug through reduction of the nitro group and result in formation of intermediate metabolites. The resultant compounds formed are highly active, bind to bacterial ribosomes and obstruct several bacterial enzymes needed for the synthesis of DNA, RNA and other metabolic enzymes. Nitrofurantoin exerts a unique type of mechanism of action and works by interfering with bacterial growth at three different sites in the Krebs's cycle. It inhibits a bunch of bacterial enzymes that participate in bacterial carbohydrate assimilation at three points in the Krebs cycle, ${ }^{5}$ and in the interfere during the process of cell wall synthesis. ${ }^{8}$ The nitro group combined with heterocyclic furan ring constitutes the active site of the drug which needs to be initiated by microbial nitro reductase enzymes for their action. ${ }^{20}$ Its action is dependent upon activation by bacterial nitroreductase flavoproteins, such as Escherichia coli nitroreductase. ${ }^{21}$

\section{DISCUSSION}

\section{Therapeutic Uses of Nitrofurantoin}

Urinary Tract Infection- Nitrofurantoin is advocated as first line therapy for uncomplicated UTIs in women. Nitrofurantoin is especially used to treat UTIs caused by Gram-negative bacteria, particularly Escherichia coli. ${ }^{22}$ Nitrofurantoin is being advised for adults and children in the therapy of acute symptomatic UTIs. Also, prescribed for the treatment of recurrent UTIs and for the prophylaxis of recurrent UTIs. ${ }^{5}$ Nitrofurantoin is beneficial for treating UTIs in older women with low glomerular filtration rates. ${ }^{23}$ Since nitrofurantoin reaches therapeutically active concentrations only in lower urinary tract, hence it is neither recommended for upper UTI treatment nor for men with UTI and concomitant prostatitis. ${ }^{24}$

Acute Uncomplicated UTI- Acute uncomplicated cystitis is one of the most common health problems in the society for which young women seek medical attention. These inflammatory diseases are most commonly caused by Escherichia coli, can be treated by number of oral antibiotics, although increasing resistance are reported to some of the commonly used antimicrobial agents, particularly trimethoprim-sulphamethoxazole (TMP-SMX) combination. In women having risk factors for infection with resistant bacteria, or in the situation of a high prevalence of TMP-SMX resistant bacteria, a fluoroquinolone or nitrofurantoin should be considered for empirical treatment. Use of nitrofurantoin does not have chances of cross-resistance. Beta-lactams and fosfomycin may be suggested as second line agents for empirical treatment of cystitis. ${ }^{25}$

Acute Cystitis- Cystitis can be defined as the inflammatory condition of the urinary bladder with various and often unknown aetiology. The most familiar pathogen in uncomplicated and complicated cystitis is uropathogen Escherichia coli strain, followed by $S$ saprophyticus, enterococci, coagulase-negative staphylococci, and other species of Enterobacteriaceae.26,27 Cystitis can be clinically reported as a syndrome of dysuria, urgency, frequency, and lower abdominal pain. While cystitis is usually occurred by bacterial infection, it can also be induced by non-infectious conditions such as carcinoma in situ, bladder cancer, and bladder stone or it can even emerge from unknown origin as in interstitial cystitis. ${ }^{26}$ Uncomplicated cystitis can be reported as an infection in women with a structurally and functionally normal urinary bladder. However, complicated cystitis is related to structurally or functionally abnormal urinary bladder, where the host cell immunity is compromised and microorganisms develop antimicrobial resistance. ${ }^{28,29}$ Classical symptoms of uncomplicated cystitis 
are acute, and include dysuria, urge, and increased urinary frequency. These symptoms give rise to a $95 \%$ post symptom probability for uncomplicated cystitis. $^{30}$ With reported research outcome data, following treatment options are available- 1. No treatment: Symptoms self-limited, ${ }^{31}$ increased fluid intake by the patient may be helpful for the recognition of the condition, and complications are rare. 2. Wait-and watch the prescription: This plan of action has resulted in decreased use of antibiotics for other infections encountered in general practice.32 3. NSAIDs used for symptom relief: Ibuprofen has shown similar efficacy to ciprofloxacin in symptomatic relief. ${ }^{33}$ 4. Three days' treatment with a suitable antimicrobial agent is recommended. ${ }^{34}$

Three days' treatment of uncomplicated cystitis has the same effect as extended course of therapy, but fewer adverse effects have been reported associated with three day's therapy. ${ }^{33}$ Nitrofurantoin should be considered because of low resistance report. Trimethoprim is as effective as cotrimoxazole in uncomplicated cystitis with fewer adverse effects. Pivmecillinam also shows favourable resistance levels. Quinolones should be restrained in case of more lifethreatening infections, and have no role in the empiric treatment of uncomplicated cystitis. The most successful therapy for an uncomplicated cystitis is a three-day course with TMP-SMX. ${ }^{35}$ There was no difference in symptom improvement between 3 days and 5-10 days of antibiotic use in women with uncomplicated cystitis, however the best therapeutic outcome from the microbiological aspect were reported with 5-10 days therapy. ${ }^{34,36}$

Pyelonephritis- In patients of uncomplicated pyelonephritis, fluoroquinolones are better to TMP-SMX for empirical therapy because of presence of TMP-SMX resistance against uropathogens causing pyelonephritis. However, TMP-SMX is a suitable option for patients with mild to moderate uncomplicated pyelonephritis, if the uropathogen is known to be susceptible to this agent. It is rational to use 7-10 days oral fluoroquinolone for outpatient management of mild to moderate pyelonephritis in the surroundings of a susceptible causative pathogen. The majority of women with acute uncomplicated pyelonephritis are managed carefully and effectively in outpatient setting. ${ }^{25}$ The frequency of acute pyelonephritis in majority of reported studies ranges from $0.5 \%$ to $2 \%$ of all pregnancies. ${ }^{37}$ The customary antibiotics used for the treatment of acute pyelonephritis include nitrofurantoin, cefazolin, cephalexin, ceftriaxone, and gentamicin. ${ }^{38}$ Nitrofurantoin or amoxicillinclavulanic acid are effective in terms of bacterial sensitivity, but nitrofurantoin should be avoided in patients with pyelonephritis, the reason being poor serum and tissue levels.

Pregnancy and Breast Feeding- Pregnancy is a special condition in which the pregnant woman undergoes significant physiological, structural and functional changes that may assist ascending infections from the urethra. UTIs are one of the most repeated complications of pregnancy. Nitrofurantoin is actively transported into breast milk, through the breast cancer resistance protein (BCRP) transporter. ${ }^{39}$ The US Food and Drug Administration put nitrofurantoin in category B drug. Nitrofurantoin can be safely used throughout the first trimester of pregnancy. Study conducted in Israel did not document any relationship between exposures of nitrofurantoin to pregnant woman with increased risk of major malformations. Further, the author reported that first trimester manifestation due to nitrofurantoin therapy was not associated with increased risk for total major congenital malformations. 40 However, an American nationwide population based reported a link between maternal use of sulfonamides and nitrofurantoin (1 month before pregnancy to the end of the first trimester) with serious birth defects. ${ }^{41} \mathrm{~A}$ research conducted in Norway reported a possible relationship between the use of nitrofurantoin in the last month of pregnancy and jaundice in new-borns. ${ }^{42}$ The authors explained that the discovery was biologically possible, since nitrofurantoin can lead to haemolytic anaemia in newborns. Drug therapy management of UTIs in pregnancy need proper diagnostic workup and thorough understanding of antimicrobial agents to enhance maternal outcome, protect safety to the fetus, and prevent complications that may lead to significant morbidity and mortality in both the fetus and the mother. ${ }^{38}$ Nitrofurantoin and TMP-SMX therapy best to be avoided during the first trimester of pregnancy because of possible risk of fetal defects. Moreover, nitrofurantoin can alter glutathione reductase activity and consequently can cause haemolytic anaemia. Newborns and fetuses are susceptible to this effect of altered glutathione reductase activity and consequently haemolytic crises may happen in these patients. ${ }^{43,44}$ Further, evidence links craniosynostosis in fetus with the exposures to nitrofurantoin and drugs with similar chemical structures. ${ }^{45,46}$

Interaction- Studies have indicated that antacid when taken with nitrofurantoin, increases the ionization of later, resulting in decreased absorption of this drug. Nitrofurantoin, a potent inhibitor of primary adenine diphosphate causes platelet aggregation in vitro. Nitrofurantoin may interfere with a number of laboratory test results. For example, in urine glucose estimation which uses Benedict's reagent may give a false-positive result in presence of nitrofurantoin. Nitrofurantoin therapy may elevate serum levels of glucose, bilirubin, alkaline phosphatase and blood urea nitrogen. ${ }^{5,47}$ Therapy nitrofurantoin may cause oxidative damage in patients with a deficiency of glucose-6-phosphate dehydrogenase enzyme. Both nitrofurantoin and phenazopyridine are capable to make appearance of a soxS': lacZ fusion. Subinhibitory concentrations of phenazopyridine results in increased therapeutic outcome of ampicillin, chloramphenicol, tetracycline, and nitrofurantoin. The expression of soxRS regulon gene appear to be a drawback for Escherichia coli during nitrofurantoin therapy; but might be an advantage during phenazopyridine therapy, particularly when the final compound could act as a selective pressure for mutations related to virulence and multi antibiotic resistance. 48 Nitrofurantoin reaction with eosinophilia produces systemic symptom, may be lifethreatening condition, although very rarely observed. ${ }^{49,50}$

Side Effects- Nitrofurantoin is normally well tolerated with common side effects include nausea and headache, Table 1. The uncommon side effects are aplastic anemia, peripheral neuropathy, liver toxicity, pulmonary toxicity, and Stevens Johnson syndrome (SJS). ${ }^{50}$ Nitrofurantoin may generate many pulmonary signs and symptoms like chronic or acute 
interstitial pneumonia, pulmonary hemorrhage, bronchoconstriction, anaphylaxis and pleural effusion. ${ }^{51}$ Nitrofurantoin associated reactions have been reported in many studies. Long established acute pulmonary response develops within hours to weeks after the consumption of the medicine. This reaction syndrome is distinguished by sudden onset of fever, chills, cough, myalgia, and dyspnoea ${ }^{47}$ Subacute pulmonary reactions due to nitrofurantoin therapy normally appear after one month of drug intake. This reaction is marked by persevering and progressive cough, dyspnea, orthopnea and fever. ${ }^{47}$ Chronic nitrofurantoin pulmonary event is another form of reaction, associated with indirect production of nonproductive cough and dyspnoea. ${ }^{52}$ Present clinical documents and health professionals ought to spotlight the possible pulmonary toxicity, while recommending this medicine. Adverse reactions, mostly related to chronic utilization of nitrofurantoin, includes gastrointestinal problems, hepatotoxicity, peripheral neuropathy, hematologic disorders, neurological defects, skin eruptions, pulmonary problems and other malformations. ${ }^{47}$ Occasionally nausea may be present, rare with macrocrystalline formulations. In case of long-term, adverse events, such as pulmonary toxicity and neuropathy induced due to nitrofurantoin therapy is important. ${ }^{17}$ During nitrofurantoin therapy acute hemolytic anemia is the major hematological adverse effect associated with deficiency of glucose-6-phosphate dehydrogenase. ${ }^{53}$ Pulmonary fibrosis and hepatotoxicity problems have been reported in patients receiving nitrofurantoin as prophylaxis for several months or years. $^{24}$ Acute pulmonary syndrome, a rare but severe adverse reaction linked to nitrofurantoin therapy. This syndrome is seldom observed at the moment of presentation, which potentially subjects patients to unnecessary treatment and result in discontinuation of nitrofurantoin therapy. ${ }^{54}$ Chronic drug induced lung disease due to nitrofurantoin therapy after long term use is noticed frequently in aged women presenting with respiratory problems. ${ }^{55}$ In normal renal function patients, the optimal level of nitrofurantoin urinary concentration is $50-300 \mathrm{mg} / \mathrm{mL}^{18}$ The therapeutic urinary concentration of nitrofurantoin cannot be achieved and may result in therapeutic failure if a creatinine clearance is $<30 \mathrm{~mL} / \mathrm{min}^{17,18}$ Hence, nitrofurantoin is not to be use in patients with a creatinine clearance $<30 \mathrm{~mL} / \mathrm{min} .{ }^{18,56}$

\begin{tabular}{|c|c|c|}
\hline Organs & Adverse Drug Reaction & Range of Reaction \\
\hline $\begin{array}{c}\text { Gastro- } \\
\text { intestinal }^{24}\end{array}$ & $\begin{array}{c}\text { Nausea, abdominal discomfort, } \\
\text { headache }\end{array}$ & Acute reaction \\
\hline Liver $^{57}$ & Hepatotoxicity, chronic hepatitis & $\begin{array}{c}\text { Acute or may be } \\
\text { chronic reaction }\end{array}$ \\
\hline Neurologic $^{58}$ & Peripheral neuropathy & Chronic reaction \\
\hline Respiratory & $\begin{array}{c}\text { Pulmonary fibrosis \& Pulmonary } \\
\text { toxicity }\end{array}$ & Chronic reaction \\
\hline $\begin{array}{c}\text { Allergic } \\
\text { reaction }\end{array}$ & $\begin{array}{c}\text { Cough, fever, chills, myalgia, } \\
\text { dyspnoea, orthopnea }\end{array}$ & $\begin{array}{c}\text { Acute, Sub-acute or } \\
\text { Chronic reaction }\end{array}$ \\
\hline Others $^{59}$ & $\begin{array}{c}\text { Haematological reaction; Example- } \\
\text { Haemolytic anaemia }\end{array}$ & Chronic reaction \\
\hline
\end{tabular}

Precautions- Various physiologic adaptations occurs with advancing gestation, including marked increase in maternal intravascular volume, glomerular filtration rate, and hepatic and metabolic activities; thinning and maturation of the fetomaternal membrane; and enlarge transplacental diffusion capacity. Because of these above special changes the maternal antibiotic concentrations tend to be $10 \%-50 \%$ lower in late pregnancy and the instant postpartum period than in the non-pregnant state. Placental transport of antibiotics and their excretion in amniotic fluid or breast milk are similarly affected by hemodynamic changes, membrane transport characteristics, and maturation or metabolic activity of the specific organs involved. 60 Because of the fact that the antibiotics administered to the mother may reach and affect the fetus, hence when prescribing antibiotics during pregnancy, one must take into thought both maternal and fetal well-being. Therapy should be selected according to the maternal disease and also be safe for the fetus. As the volume of distribution is increased during pregnancy, so the dosages may need to be adjusted to accommodate this change. Because it is hard to monitor the toxic effect to the fetus, the physician must build careful choices when considering antibiotics for the pregnant woman. ${ }^{61}$ As uninfected women frequently experience symptoms associated with UTIs and many bacteriuric women are asymptomatic, all pregnant women should be recommended for quantitative urine cultures because bacteriuria during pregnancy may produce serious complications, such as development of acute pyelonephritis in the third trimester and premature labour. The American Geriatrics Society 2015 Beers Criteria has improved Expert Panel and has revised its guidance to avoid use of nitrofurantoin in renal impairment in patients with a creatinine clearance (Clcr) less than $60 \mathrm{~mL} / \mathrm{min}$ to those with a Clcr of less than $30 \mathrm{~mL} / \mathrm{min}$, after the findings of two retrospective studies that identified the safety and benefit of nitrofurantoin in that population. This change in guidance, along with increasing resistance to both sulfamethoxazoletrimethoprim and fluoroquinolones, gave rise to the advantages of nitrofurantoin and suggested for the consideration of nitrofurantoin when prescribing to the elderly patients. ${ }^{62}$

\section{Antimicrobial Resistance of Nitrofurantoin}

Infections caused by antibiotic resistant pathogens are of great concern which is associated with higher mortality and morbidity. ${ }^{63}$ In UTI patients, bacterial resistance rates to frequently prescribe antibiotics are continually jumping. Surprisingly, in many countries $>20 \%$ of microorganisms causing UTI is resistant to TMP-SMX and cephalosporins. Increasing rates of resistance is also being noticed to fluoroquinolones with resistance rates up to $10 \% \cdot 64,65$ In a surveys conducted in USA and Canada among extended spectrum beta lactamase (ESBL) producing isolates the rate of resistance to nitrofurantoin were $1.1 \%$, in a total of 1,142 isolates of Escherichia coli from a outpatient urinary department. 66 Almost same findings were documented in France, where, $1.8 \%$ of all urinary Escherichia coli isolates were found to be resistant to nitrofurantoin. ${ }^{67}$ High sensitivity rates of $88 \%$ and $90 \%$, against ESBL isolates, were reported by Pai et al. and Sire et al., respectively. ${ }^{68}$ A survey based study conducted in hospitals of Latin America and Italy reported high rates of resistance in Escherichia coli to nitrofurantoin. ${ }^{69} \mathrm{~A}$ study conducted in a teaching hospital in Taiwan reported high susceptibility rate (65\%) of Escherichia coli among the ESBL isolates to nitrofurantoin..$^{70}$ In Europe, varying rates of resistance to nitrofurantoin is noticed, on an average, $1.2 \%$ of the cases, where the rates of resistance were highest in Portugal and Spain, 5.8\%, 4.2\%), respectively and 
lowest in Finland $(0.5 \%)$ and virtually no resistance in Sweden. ${ }^{71}$ Very recent study conducted in an outpatient department in USA documented a very low (<3\%) Escherichia coli resistance to nitrofurantoin. ${ }^{72}$ Novel resistance mechanisms exhibited by bacteria against nitrofurantoin is presented in Table 2. Nitrofurantoin is bactericidal in nature, nonspecific molecular target, and limited exposure beyond urinary tract may have the advantage with low resistance rate seen with this antibiotic. ${ }^{22}$ Nitrofurantoin has remained successful after many decades of extensive use worldwide with very low resistance rate.

\begin{tabular}{|c|c|c|}
\hline Bacteria & $\begin{array}{c}\text { Antibiotic } \\
\text { Resistance }\end{array}$ & Mechanism of Resistance \\
\hline Escherichia coli $^{73}$ & Nitrofurantoin & $\begin{array}{c}\text { Mutation in nsfA and } \\
\text { nfsB genes }\end{array}$ \\
\hline Escherichia coli $^{74}$ & Nitrofurantoin & Plasmid mediated OqxAB \\
\hline Enterobacteriaceae $^{75}$ & Nitrofurantoin & $\begin{array}{c}\text { albeit oqxAB prevalence, } \\
\text { Mutation in nfsA, nfsB and ribE } \\
\text { proteins }\end{array}$ \\
\hline Klebsiella pneumoniae $^{76}$ & Nitrofurantoin & ESBL production $(24 \%)$ \\
\hline${\text { Citrobacter } \text { species }^{77}}^{\text {Nitrofurantoin }}$ & ESBL production \\
\hline
\end{tabular}

\section{New Applications of Nitrofurantoin}

In the light of growing reports of antibiotic resistance coupled with multidrug resistance organisms, there is a pressing need of newer and effective agents for complete cure of UTI. Recent research has suggested nitrofurantoin as the treatment choice for UTI caused by multi drug resistant bacteria. In this regard, nitrofurantoin is vital force presently used to treat vancomycin resistant enterococci (VRE) and nosocomial UTIs (Catheter-associated). Nitrofurantoin is active against both vancomycin sensitive enterococci (VSE) and VRE. Oral nitrofurantoin therapy is recommended for nosocomial VSE or VRE catheter-associated bacteriuria. ${ }^{19}$ Recent research outcome data suggested nitrofurantoin as an alternative for extended spectrum beta lactamase (ESBL) producing Escherichia coli.78 Furthermore, several studies have indicated nitrofurantoin as an alternative for the therapy of ESBL producing Escherichia coli related lower UTI. ${ }^{78,79,80}$ An Europe based surveillance study under Antimicrobial Resistance Epidemiology Survey on Cystitis project suggested fosfomycin tromethamine, nitrofurantoin and pivmecillinam as effective agents for the treatment of uncomplicated UTIs. 81

\section{CONCLUSIONS}

Nitrofurantoin achieves therapeutic concentrations only in lower urinary tract. This limits its use to the treatment of lower UTI. Nitrofurantoin is highly active against Escherichia coli and $S$ saprophyticus isolates. The toxicity of short course of therapy with nitrofurantoin is mild and predominantly gastrointestinal. Nitrofurantoin had similar effectiveness when compared to other antibiotics indicated for the treatment of UTI patients, but has a greater risk of adverse events than other prophylactic treatment regimens. However, the disclosure of antibiotic resistance and the decrease in recently developed antibiotics have led to an increasing interest in the treatment and prophylaxis of bacterial UTI with nitrofurantoin. The safety profile and resistance to this antibiotic remained practically unchanged since its marketing. In this changing trend of microorganisms coupled with multidrug resistant bacteria, the role of nitrofurantoin is very crucial, particularly in cases of ESBL producing enteric bacteria, VSE and VRE.

\section{REFERENCES}

[1] Flores-Mireles AL, Walker JN, Caparon M, et al. Urinary tract infection: epidemiology, mechanisms of infection and treatment options. Nat Rev Microbial 2015;13(5):269-84.

[2] Jhang JF, Kuo HC. Recent advances in recurrent urinary tract infection from pathogenesis and biomarkers to prevention. Ci Ji Yi Xue Za Zhi 2017;29(3):131-7.

[3] Lane DR, Takhar SS. Diagnosis and management of urinary tract infection and pyelonephritis. Emergency Medicine Clinics of North America 2011;29(3):539-52.

[4] Ronald A. The etiology of urinary tract infection: traditional and emerging pathogens. Dis Mon 2003;49(2):71-82.

[5] Cunha BA. Nitrofurantoin: an update. Obstet Gynecol Surv 1989;44(5):399-406.

[6] Muller AE, Verhaegh EM, Harbarth S, et al. Nitrofurantoin's efficacy and safety as prophylaxis for urinary tract infections: a systematic review of the literature and meta-analysis of controlled trials. Clinical Microbiology and Infection 2017;23(6):355-62.

[7] Guay DR. An update on the role of nitrofurans in the management of urinary tract infections. Drugs 2001;61(3):353-64.

[8] Gleckman R, Alvarez S, Joubert DW. Drug therapy reviews: Nitrofurantoin. Am J Hosp Pharm 1979;36(3):342-51.

[9] Mavromanolakis E, Maraki S, Samonis G, et al. Effect of norfloxacin, trimethoprim-sulfamethoxazole and nitrofurantoin on fecal flora of women with recurrent urinary tract infections. J Chemother 1997;9(3):203-7.

[10] Conklin JD. The pharmacokinetics of nitrofurantoin and its related bioavailability. Antibiot Chemother 1978;25:233-52.

[11] Ben-Noun L. Drug-induced respiratory disorders: incidence, prevention and management. Drug saf 2000;23(2):143-64.

[12] Gupta K, Hooton TM, Stamm WE. Increasing antimicrobial resistance and the management of uncomplicated community-acquired urinary tract infections. Ann Intern Med 2001;135(1):41-50.

[13] Oplinger M, Andrews CO. Nitrofurantoin contraindication in patients with a creatinine clearance below $60 \mathrm{~mL} / \mathrm{min}$ : Looking for the evidence. Ann Pharmacother 2013;47(1):106-11.

[14] Lindgren $\mathrm{KP}$, Klockars $\mathrm{O}$, Malmberg $\mathrm{C}$, et al. Pharmacodynamic studies of nitrofurantoin against common uropathogens. J Antimicrobial Chemother 2015;70(4):1076-82.

[15] Adkison KK, Vaidya SS, Lee DY, et al. The ABCG2 C421A polymorphism does not affect oral nitrofurantoin pharmacokinetics in healthy Chinese male subjects. $\mathrm{Br} \mathrm{J}$ Clin Pharmacol 2008;66(2):233-9. 
[16] Gilbert DN. Urinary tract infections in patients with chronic renal insufficiency. Clin J Am Soc Nephrol 2006;1(2):327-31.

[17] Brumfitt W, Hamilton-Miller JM. Efficacy and safety profile of long-term nitrofurantoin in urinary infections: 18 years' experience. J Antimicrob Chemother 1998;42(3):363-71.

[18] Grayson ML, Whitby M. Nitrofurans: nitrofurazone, furazolidone and nirofurantoin. The use of antibiotics. $6^{\text {th }}$ edn. London, England: Hodder Arnold 2010: p. 1195204.

[19] Cunha BA. New uses for older antibiotics: nitrofurantoin, amikacin, colistin, polymyxin B, doxycycline and minocycline revisited. Med Clin North Am 2006;90(6):1089-107.

[20] Koulaouzidis A, Bhat S, Moschos J, et al. Nitrofurantoin induced lung- and hepatotoxicity. Ann Hepatol 2007;6(2):119-21.

[21] Race PR, Lovering AL, Green RM, et al. Structural and mechanistic studies of Escherichia coli nitroreductase with the antibiotic nitrofurazone. Reversed binding orientations in different redox states of the enzyme. J Biol Chem 2005;280(14):13256-64.

[22] Ingalsbe ML, Wojciechowski AL, Smith KA, et al.. Effectiveness and safety of nitrofurantoin in outpatient male veterans. Ther Adv Urol 2015;7(4):186-93.

[23] Singh N, Gandhi S, McArthur E, et al. Kidney function and the use of nitrofurantoin to treat urinary tract infections in older women. CMAJ 2015;187(9):648-56.

[24] Huttner A, Verhaegh EM, Harbarth S, et al. Nitrofurantoin revisited: a systematic review and meta-analysis of controlled trials. J Antimicrob Chemother 2015;70(9):2456-64.

[25] Hooton TM. Fluoroquinolones and resistance in the treatment of uncomplicated urinary tract infection. Int J Antimicrob Agents 2003;22(Suppl 2):65-72.

[26] Dobrindt U, Hacker J. Uropathogenes and virulence factors. In: Naber KG, Schaeffer AJ, Heyns CF, et al. eds. Urogenital infections. Arnhem, The Netherlands: European Association of Urology 2010: p. 4-22.

[27] Schaeffer AJ, Schaeffer EM. Infections of the urinary tract. In: AJ Wein, LR Kavoussi, AC Novick, et al. eds. CampbellWalsh Urology. $10^{\text {th }}$ edn. Philadelphia: Elsevier Saunders 2012;1:257-326.

[28] Sundqvist M, Kahlmeter G. Uncomplicated and community acquired urinary tract infections: aetiology and resistance. The Netherlands: European Association of Urology 2010: p. 72-81.

[29] Bader MS, Hawboldt J, Brooks A. Management of complicated urinary tract infections in the era of antimicrobial resistance. Postgraduate Med 2010;122(6):7-15.

[30] Bent S, Nallamothu BK, Simel DL, et al. Does this woman have an acute uncomplicated urinary tract infection? JAMA 2002;287(20):2701-10.

[31] Christiaens TCM, De Meyere M, Verschraegen G, et al. Randomised controlled trial of nitrofurantoin versus placebo in the treatment of uncomplicated urinary tract infection in adult women. $\mathrm{Br} J$ Gen Pract 2002;52(482):729-34.
[32] Hoye S, Frich JC, Lindbaek M. Use and feasibility of delayed prescribing for respiratory tract infections: a questionnaire survey. BMC Fam Pract 2011;12:34.

[33] Bleidorn J, Gagyor I, Kochen MM, et al. Symptomatic treatment (ibuprofen) or antibiotics (ciprofloxacin) for urinary tract infection? Results from a randomized controlled pilot trial. BMC Med 2010;8:30.

[34] Milo G, Katchman EA, Paul M, et al. Duration of antibacterial treatment for uncomplicated urinary tract infection in women. Cochrane database Syst Rev 2005;(2):CD004682.

[35] Orenstein R, Wong ES. Urinary tract infections in adults. Am Fam Physician 1999;59(5):1225-34, 1237.

[36] Talan DA, Stamm WE, Hooton TM, et al. Comparison of ciprofloxacin (7 days) and trimethoprimsulfamethoxazole (14 days) for acute uncomplicated pyelonephritis pyelonephritis in women: a randomized trial. JAMA 2000;283(12):1583-90.

[37] Schnarr J, Smaill F. Asymptomatic bacteriuria and symptomatic urinary tract infection in pregnancy. Eur J Clin Invest 2008;38(Suppl 2):50-7.

[38] Le J, Briggs GG, McKeown A, et al. Urinary tract infections during pregnancy. Ann Pharmacother 2004;38(10):1692-701.

[39] Merino G, Jonker JW, Wagenaar E, et al. The breast cancer resistance protein (BCRP/ABCG2) affects pharmacokinetics, hepatobiliary excretion and milk secretion of the antibiotic nitrofurantoin. Mol Pharmacol 2005;67(5):1758-64.

[40] Goldberg O, Koren G, Landau D, et al. Exposure to nitrofurantoin during the first trimester of pregnancy and the risk for major malformations. J Clin Pharmacol 2013;53(9):991-5.

[41] Crider KS, Cleves MA, Reefhuis J, et al. Antibacterial medication use during pregnancy and risk of birth defects: National Birth Defects Prevention Study. Arch Pediatr Adolesc Med 2009;163(11):978-85.

[42] Nordeng H, Lupattelli A, Romøren M, et al. Neonatal outcomes after gestational exposure to nitrofurantoin. Obstet Gynecol 2013;121(2 Pt 1):306-13.

[43] Gait JE. Hemolytic reactions to nitrofurantoin in patients with glucose-6-phosphate dehydrogenase deficiency: theory and practice. DICP 1990;24(12):1210-3.

[44] Bruel H, Guillemant V, Saladin-Thiron C, et al. Anemie hemolytique chez un nouveau-ne apres prise maternelle de nitrofuratoïne en fin de grossesse. Arch Pediatr 2000;7:745-7.

[45] Gardner JS, Guyard-Boileau B, Alderman BW, et al. Maternal exposure to prescription and non- prescription pharmaceuticals or drugs of abuse and risk of craniosynostosis. Int J Epidemiol 1998;27(1):64-7.

[46] Kallen B, Robert-Gnansia E. Maternal drug use, fertility problems and infant craniostenosis. Cleft Palate Craniofac J 2005;42(6):589-93.

[47] Munoz-Davila MJ. Role of old antibiotics in the era of antibiotic resistance. Highlighted nitrofurantoin for the treatment of lower urinary tract infections. Antibiotics 2014;3(1):39-48.

[48] Amábile-Cuevas CF, Arredondo-García JL. Nitrofurantoin, phenazopyridine and the superoxide-response regulon soxRS of Escherichia coli. J Infect Chemother 2013;19(6):1135-40. 
[49] Velema MS, Voerman HJ. Dress syndrome caused by nitrofurantoin. Neth J Med 2009;67(4):147-9.

[50] Leao RN, Barreto P, Leao RR, et al. Nitrofurantoin: Cause of dress syndrome. BMJ Case Rep 2013;2013.pii: bcr2013008991.

[51] Travis WD, Colby TV, Koss MN, et al. Non-neoplastic disorders of the lower respiratory tract. Atlas of nontumor pathology. Washington, The American Registry of Pathology 2002;2:327.

[52] Marshall AD, Dempsey OJ. Is "nitrofurantoin lung" on the increase? Br Med J 2013;346:f3897.

[53] Holmberg L, Boman G, Bottiger LE, et al. Adverse reactions to nitrofurantoin. An analysis of 921 reports. Am J Med 1980;69(5):733-8.

[54] Broes MJ, Roelofs BF, Mudde AH, et al. Pulmonary toxicity resulting from the use of nitrofurantoin. Ned Tisdschr Geneeskd 2012;156(44):A4990.

[55] Mendez JL, Nadrous HF, Hartman TE, et al. Chronic nitrofurantoin-induced lung disease. Mayo Clin Proc 2005;80(10):1298-302.

[56] Cunha BA. Nitrofurantoin: current concepts. Urology 1988;32(1):67-71.

[57] Koulaouzidis A, Bhat S, Moschos J, et al. Nitrofurantoininduced lung- and hepatotoxicity. Ann Hepatol 2007;6(2):119-21.

[58] Tan IL, Polydefkis MJ, Ebenezer GJ, et al. Peripheral nerve toxic effects of nitrofurantoin. Arch Neurol 2012;69(2):265-8.

[59] Kiang TK, Ford JA, Yoshida EM, et al. Nitrofurantoinassociated lung and liver toxicity leading to liver transplantation in a middle-aged patient. Can J Hosp Pharm 2011;64(4):262-70.

[60] Chow AW, Jewesson PJ. Pharmacokinetics and safety of antimicrobial agents during pregnancy. Rev Infect Dis 1985;7(3):287-313.

[61] Hedstrom S, Martens MG. Antibiotics in pregnancy. Clin Obstet Gynecol 1993;36(4):886-92.

[62] Hoang P, Salbu RL. Updated nitrofurantoin recommendations in the elderly: a closer look at the evidence. Consult Pharm 2016;31(7):381-4.

[63] Shakti L, Veeraraghavan B. Advantage and limitations of nitrofurantoin in multi-drug resistant Indian scenario. Indian J Med Microbiol 2015;33(4):477-81.

[64] De Backer D, Christiaens T, Heytens S, et al. Evolution of bacterial susceptibility pattern of Escherichia coli in uncomplicated urinary tract infections in a country with high antibiotic consumption: a comparison of two surveys with a 10 year interval. J Antimicrob Chemother 2008;62(2):364-8.

[65] Schito GC, Naber KG, Botto H, et al. The ARESC study: an international survey on the antimicrobial resistance of pathogens involved in uncomplicated urinary tract infections. Int J Antimicrob Agents 2009;34(5):407-13.

[66] Zhanel GG, Hisanaga TL, Laing NM, et al. Antibiotic resistance in Escherichia coli outpatient urinary isolates: Final results from the North American Urinary Tract Infection Collaborative Alliance (NAUTICA). Int J Antimicrob Agents 2006;27(6):468-75.

[67] Honderlick P, Cahen P, Gravisse J, et al. Quelle sensibilite aux antibiotiques pour les bactéries responsables d'infections urinaires? Que penser de fosfomycine et nitrofuranes? Pathol Biol 2006;54(8-9):462-6.
[68] Pai AK, Rai R, Sanjeev $H$, et al. Nitrofurantoin: an alternative therapy for uncomplicated cystitis in the era of antimicrobial resistance. J Clin Diagn Res 2011;5(5):964-6.

[69] Fadda G, Nicoletti G, Schito GC, et al. Antimicrobial susceptibility patterns of contemporary pathogens from uncomplicated urinary tract infection isolated in a multicenter Italian survey: possible impact in guidelines. J Chemother 2005;17(3):251-7.

[70] Liu HY, Lin HC, Lin YC, et al. Antimicrobial susceptibilities of urinary extended spectrum betalactamase-producing Escherichia coli and Klebsiella pneumoniae to fosfomycin and nitrofurantoin in a teaching hospital in Taiwan. J Microbiol Immunol Infect 2011;44(5):364-8.

[71] Kahlmeter G. The ECO.SENS Project: a prospective, multinational, multicentre epidemiological survey of the prevalence and antimicrobial susceptibility of urinary tract pathogens-interim report. J Antimicrob Chemother 2000;46(Supppl 1):15-22.

[72] Sanchez GV, Babiker A, Master RN, et al. Antibiotic resistance among urinary isolates from female outpatients in the united states in 2003 and 2012. Antimicrob Agents Chemother 2016;60(5):2680-3.

[73] Sandegren L, Lindquist A, Kahlmeter G, et al. Nitrofurantoin resistance mechanism and fitness cost in Escherichia coli. J Antimicrob Chemother 2008;62(3):495-503.

[74] Ho PL, Ng KY, Lo W, et al. Plasmid-mediated OqxAB is an important mechanism for nitrofurantoin resistance in Escherichia coli. Antimicrob Agents Chemother 2016;60(1):537-43.

[75] Sekyere OJ. Genomic insights into nitrofurantoin resistance mechanisms and epidemiology in clinical Enterobacteriaceae. Future Sci OA 2018;4(5):FSO293.

[76] El Bouamria MC, Arsalanea L, El Kamounia Y, et al. Antimicrobial susceptibility of urinary Klebsiellapneumoniae and the emergence of carbapenem-resistant strains: a retrospective study from a university hospital in Morocco, North Africa. African J Urology 2015;21(1):36-40.

[77] Sami H, Sultan A, Rizvi M, et al. Citrobacter as auropathogen, its prevalence and antibiotics susceptibility pattern. CHRISMED J Health Res 2017;4(1):23-6.

[78] Garau J. Other antimicrobials of interest in the era of extended-spectrum beta-lactamases: fosfomycin, nitrofurantoin and tigecycline. Clin Microbiol Infect 2008;14(Suppl 1):198-202.

[79] Tasbakan MI, Pullukcu H, Sipahi OR, et al. Nitrofurantoin in the treatment of extended-spectrum $\beta$-lactamaseproducing Escherichia coli-related lower urinary tract infection. Int J Antimicrob Agents 2012;40(6):554-6.

[80] Chen YH, Ko WC, Hsueh PR. Emerging resistance problems and future perspectives in pharmacotherapy for complicated urinary tract infections. Expert Opin Pharmacother 2013;14(5):587-96.

[81] Naber KG, Schito G, Botto H, et al. Surveillance study in Europe and Brazil on clinical aspects and Antimicrobial Resistance Epidemiology in Females with Cystitis (ARESC): implications for empiric therapy. Eur Urol 2008;54(5):1164-75. 